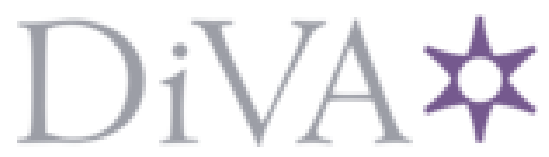

http://www.diva-portal.org

\title{
Postprint
}

This is the accepted version of a paper published in Acta Obstetricia et Gynecologica Scandinavica. This paper has been peer-reviewed but does not include the final publisher proof-corrections or journal pagination.

Citation for the original published paper (version of record):

Niklasson, B., Börjesson, A., Carmnes, U., Segerdahl, M., Georgsson Öhman, S. et al. (2012)

Intraoperative injection of bupivacaine-adrenaline close to the fascia reduces morphine requirements after cesarean section: a randomized controlled trial.

Acta Obstetricia et Gynecologica Scandinavica, 91(12): 1433-1439

http://dx.doi.org/10.1111/j.1600-0412.2012.01480.x

Access to the published version may require subscription.

N.B. When citing this work, cite the original published paper.

Permanent link to this version:

http://urn.kb.se/resolve?urn=urn:nbn:se:shh:diva-1205 


\section{Intraoperative injection of bupivacaine-adrenaline close to the fascia reduces morphine requirements after caesarean section: A randomized controlled trial}

Running title: Local anesthesia and cesarean section

Boel Niklasson"12, RN, RM, Astrid Börjesson'1, RN, RM,

Ulla-Britt Carmnes' ${ }^{1}$ RN, RM, MNSci, Märta Segerdahl³, MD, PhD,

Susanne Georgsson Öhman ${ }^{4,5}$, RN, RM, PhD, Agneta Blanck ${ }^{122}$, MD, PhD

${ }^{1}$ Department of Obstetrics and Gynecology, Karolinska University Hospital, Stockholm, Sweden

${ }^{2}$ Dept. of Clinical Science, Intervention and Technology, Karolinska Institute, Karolinska University Hospital, Stockholm, Sweden

${ }^{3}$ Department of Physiology and Pharmacology, Karolinska Institute, Stockholm, Sweden

${ }^{4}$ Sophiahemmet University College, Stockholm,

${ }^{5}$ Department of Women's and Children's health, Karolinska Institute, Stockholm, Sweden

Corresponding Author: Boel Niklasson, RM, Department of Obstetrics and Gynecology, Karolinska University Hospital, Huddinge, 14186 Stockholm, Sweden. +46-8-585 80277, boel.niklasson@ki.se 


\section{Conflict of interest}

MS is an employee at AstraZeneca who manufactures the local anesthetic, used for subcutaneous injection. No financial support for the study was received from Astra Zeneca. No potential conflicts of interest could be identified for any of the other authors. 


\section{Abstract}

Objective. The purpose of this study was to investigate whether a single injection of bupivacaine with adrenaline close to the fascia could decrease opiate consumption and pain in patients undergoing cesarean section in spinal anesthesia. Design. Randomized double-blind controlled study. Settings. Karolinska University Hospital, Huddinge, Sweden. Population. Two hundred and sixty (260) women scheduled for elective cesarean section were enrolled in the study. Methods. The treatment group $(\mathrm{n}=130)$ received $40 \mathrm{ml}$ bupivacaine $(2.5 \mathrm{mg} / \mathrm{ml})$ with adrenaline $(5 \mu \mathrm{g} / \mathrm{ml})$ (Marcain ${ }^{\circledR}$ adrenalin) and the control group $(\mathrm{n}=130)$ received $40 \mathrm{ml}$ saline solution $(0.9 \%)$, which was, in both groups, injected close to the fascia before closure of the wound. Main Outcome Measures. Morphine consumption and mean resting pain intensity Numerical Rating Scale at 12 and 24 hours were the primary outcome variables. Other assessments for pain as well as mobilization parameters were considered secondary. Results. Morphine requirements were significantly less in the bupivacaine group, 19.0 $\mathrm{mg} /$ patient, compared to $24.0 \mathrm{mg} /$ patient in the placebo group, during the first twelve postoperative hours. During this time period there was also a trend towards a difference between groups in mean pain intensity, but significant only during the first 6 hours. Over the whole first postoperative 24 hours, there were no differences in either morphine requirement or pain intensity between groups. Conclusions. A single injection of bupivacaine with adrenaline in the surgical wound decreases the need for morphine requirements for the first 12 postoperative hours and contributes to safe and effective pain management in women undergoing cesarean section. 


\section{Keywords}

Pain management, local anesthesia, bupivacaine, cesarean section, morphine consumption

\section{Abbreviations}

BMI, body mass index, CS, cesarean section, i.v., intravenous, NRS, numerical rating scale, PCA, patient controlled analgesia.

\section{Key Message box}

A single injection of bupivacaine with adrenaline in the surgical wound decreases the need for rescue morphine postoperatively and is safe and effective for pain management in women undergoing cesarean section under spinal anesthesia. 


\section{Introduction}

A significant proportion of women giving birth undergo acute or planned cesarean section (CS). The cesarean rate worldwide continues to rise and pain management after CS is an important topic. Several reports indicate that pain management is insufficient among $50-70 \%$ of the patients undergoing all types of surgery and a large number of factors have been shown to contribute (1-8). Inadequate pain control following CS has been shown to be a risk factor for a negative birth experience (9). Good pain management is strongly associated with early mobilization as well as decreased risk for complications and patient satisfaction $(8,10)$. A main difference between patients undergoing CS and patients undergoing abdominal surgery for other reasons is that the former is expected to immediately take care of a newborn baby. It is of paramount importance to optimize post-cesarean pain management to avoid disturbed bonding and interaction between mother and child $(8,9,11-13)$. Sufficient pain control is essential for successful breastfeeding, early mobilization and to shorten the time until discharge from the hospital. One third of the women in a study by Karlström et al. believed that their ability to breastfeed was affected negatively by postoperative pain (9). Any medication given to this group of patients should therefore be effective, not limit the mother's care of her newborn baby and ideally free from side-effects both in the mother and her newborn child (12).

Postoperative pain after CS consists of somatic pain mainly from the wound but also visceral pain from the uterine contractions. Different mechanisms are behind these two types of pain, and a multimodal approach to pain management is of importance $(8,14)$. One simple, but resource requiring way to approach the postoperative pain is to combine intravenous morphine with local anesthesia in the wound infiltration by continues infusion $(8,11)$.

Even though efficient postoperative pain relief can be obtained by advanced techniques like patient controlled analgesia (PCA), morphine pumps and accompanying devices may 
themselves intervene with the mobilization of the patient and the mother and child interaction. Thus, a less invasive technique with comparable efficacy, minimizing disruption of normal mother and child interaction is an advantage.

A single local injection in the surgical wound is a simple and cheap way for post surgical pain management and can be used worldwide, even in countries with limited resources for health care. Few studies address the utility of local techniques from an effectiveness perspective, i.e. how useful is the methodology in everyday clinical practice. The present study sets out to investigate the effects of a single injection of bupivacaine with adrenaline close to the fascia on opiate consumption and pain in patients undergoing CS in spinal anesthesia in a University Hospital setting. 


\section{Material and Methods}

Six-hundred and eighty-four consecutive patients scheduled for cesarean section were screened for eligibility and 260 healthy women who met the inclusion criteria were enrolled in the study. Women were included from September 2006 to April 2008 at the obstetric unit, Karolinska University Hospital, Huddinge.

Healthy women, 18-50 years old, having a planned CS from 38 full weeks of gestation were included. For practical reasons, the women had to understand the Swedish language even though some of them were born in other countries. Exclusion criteria were ongoing treatment for chronic pain, history of narcotic abuse, severe psychiatric history and any intolerance against opioids, local anesthetics or other analgesic drugs given in the study.

Women were asked to participate in the study when they visited the clinic for a preoperative assessment the day before the CS and gave their verbal and written informed consent to participate in the study. The study protocol was approved by the Regional Ethics Committee in Stockholm, Sweden (2006/628-31/1), and the Swedish Medical Products Agency (151:2006/30029). The study has been registered in the Karolinska Clinical Trial Registration database, number CT20110078. After inclusion in the study, women were randomized by using a computer based program. Randomization was performed in blocks of twenty to ensure that the same number of participants would be allocated to receive either of the two study treatments. Each randomization number had a corresponding sealed-opaque envelope, containing information of the study treatment. Envelopes were drawn sequentially from a locked box for each patient. The envelope was then opened by the nurse in the operating theatre, who also prepared the blinded syringes with the study drug before other staff involved in the procedures entered the operating room. The nurse was in no other way involved in the study. The obstetrician performing the CS received the filled anonymous syringe and 
administered the blinded injection. Forty $\mathrm{ml}$ of bupivacaine $(2.5 \mathrm{mg} / \mathrm{ml})$ with adrenaline $(5$ $\mu \mathrm{g} / \mathrm{ml}$ ) (Marcain®adrenalin) or $40 \mathrm{ml}$ saline solution (0.9\%) was administered with an injection needle reaching both the fascia and immediately above the fascia in the subcutaneous fat before closure of the wound. The women and all members of the staff responsible for postoperative care were blinded to the study treatment.

Morphine consumption was the primary outcome. Pain intensity, assessed by Numerical Rating Scale (NRS) (a numeric $10 \mathrm{~cm}$ scale $0-10$ where 0 is "no pain" at all and 10 "worst pain") and parameters related to mobilization of the women, were considered secondary outcome variables. Spinal anesthesia administered through a 27G Sproutte spinal needle was inserted at L2-L3 or at L3-L4 with the woman in a sitting position. The patients received 1.8$2.6 \mathrm{ml}$ (body height depending) intrathecal bupivacaine (Marcain Tung ${ }^{\circledR}, 5 \mathrm{mg} / \mathrm{ml}$ ), followed by $0.3 \mathrm{ml}$ fentanyl (Fentanyl ${ }^{\circledR}, 50 \mu \mathrm{g} / \mathrm{ml}$ ). During the study period, the protocol for pain treatment of women undergoing caesarean section was: Paracetamol (Perfalgan®, BristolMyers-Squibb,10 mg/ml, $100 \mathrm{ml}$ ) as a single intravenous dose after the delivery of the baby and thereafter oral administration, 1g every 6th hour, (Alvedon, AstraZeneca) combined with intravenous rescue morphine $1.0 \mathrm{mg} / \mathrm{ml}$ (Morfin MEDA®; 10mg/ml, $1 \mathrm{ml}$ diluted with $9 \mathrm{ml}$ $0.9 \%$ saline solution) until NRS estimation was at/or below 3 for the first 24 hours after the operation. After the first 24 hours morphine was substituted by oral codeine, (Kodein Recip, Recip, Sweden) 75 mg every 6th hour. Oral ibuprofen 400 mg (Brufen®, Abbott Laboratories) was given as a first dose six hours after the operation and thereafter $200 \mathrm{mg}$ every sixth hour. Oral paraffine emulsion $(30 \mathrm{ml})$ was given twice daily to diminish constipation. After surgery, when the woman arrived in the recovery room, a midwife performed uterus palpation. At this time point (about 30 minutes after closure of the wound), pain was measured for the first time by NRS. Pain at rest was then assessed every hour for the first six postoperative hours. When the woman reported NRS of 4 or above she received 
intravenous morphine from the midwife until pain intensity was NRS of 3 or below. The morphine was titrated and injected slowly by the responsible midwife. Pain evaluation was performed both at the regular time points for pain assessment and when the woman asked for morphine as rescue medication. From six hours after surgery and onwards, the evaluation of pain at rest was done every fourth hour up to 24 hours after surgery. Subsequently, NRS evaluation was done four times a day until discharge from hospital. The amount of morphine was recorded separately and accumulated and analyzed for the first 6, 12 and 24 hours. Postoperative mobilization was defined as the time points when the woman was standing next to the bed and when walking around in the room for the first time. As a measurement of the mobilization it was also noted when the woman was ready to be discharged from hospital. The wound was inspected every day, starting the day after the CS. All signs of local infections were recorded and treated according to clinical routines. When discharged from hospital, the women were prescribed oral medication with paracetamol (Alvedon, AstraZeneca), codeine, (Kodein Recip, Recip, Sweden) and ibuprofen 400 mg (Brufen®, Abbott Laboratories) for a few days, if needed. A follow-up telephone interview about pain and wound infections was performed ten days after surgery. All patients were encouraged to contact investigators if any problem came up after the Postoperative Day 10 telephone interview. Only occasional phone calls regarding pain medication were received. Most of the medical data, except the pharmaceutical records, was collected from the computer based patient record system Obstetrix $^{\mathrm{TM}}$. Data concerning administration of drugs were secured from the computer based patient chart system Take Care ${ }^{\mathrm{TM}}$. The primary data collected included: woman's age, weight and Body Mass Index (BMI) at the time of surgery, reason for performing the CS, parity and number of previous CS. The women's data were codified before they were added to the computer program IBM SPSS Statistics, version 18.0 for Windows. 


\section{Statistical analysis}

The statistic program, IBM PASW Statistics, version 18.0, was used to conduct the analyzes between the two groups. The Mann-Whitney U test was used to analyze demographic data and two tailed Student's $t$-test to analyze morphine consumption and pain assessment by Numerical Rating Scale. The level of $p \leq 0.05$ was considered significant. The study sample size estimation was based on a difference in the primary variable, morphine consumption at 0 12 and $0-24$ hrs based on reduction in morphine requirement by $25 \%$ estimated to be of magnitude to be of clinical relevance. Pain intensity, assessed as mean NRS (0-10) for the same time periods, were co-primary variables, both variables at each time interval to be in the same direction to constitute a positive outcome. Estimates of morphine requirements were based on available literature data (15). Estimates on variability in NRS were based on literature data. Co-primaries at the two different time points for both outcome variables, with a total alpha of 0.05 and a power of $80 \%$ yields, with a Bonferroni correction for multiplicity, gave a sample size estimate of 115 patients per treatment group. In order to compensate for withdrawals, 130 patients per arm were recruited 


\section{Results}

There were no significant differences between the bupivacaine group and the placebo group in patient demographics concerning BMI, age, gravidity or parity which indicates a successful randomization (Table 1).

There was significantly less morphine required in the bupivacaine group, compared to the placebo group, at 0-12 hours ( $p \leq 0.001)$, but not at 0-24 hours. Also when comparing the two groups at 0-6 hours the average bupivacaine consumption to reach postoperative pain relief was lower than in the control group $(p \leq 0.001)$. Data are presented in Figure 2.

There was no significant difference in mean pain intensity (NRS) between groups during 0-12 hours, [bupivacaine 3.96 (SD 1.57) and placebo 4.27 (SD 1.38)], or during 0-24 hours [bupivacaine 4.49 (SD 1.31) and placebo 4.54 (SD 1.21)], respectively. In the immediate postoperative period, 0-6 hours, there were lower mean and maximum pain scores by NRS, between the bupivacaine and placebo groups $(p \leq 0.001)$. Mean score is based on the investigator-analysis and represents the mean score of all recorded pain scores for a woman in any given time period (Figures 3 and 4).

When analyzing the number of requests for rescue opioids during the first six hours, 47 women in the placebo group needed five injections or more, compared to 21 women in the bupivacaine group. Thirteen women in the bupivacaine group compared to three in the placebo group never asked for any rescue morphine at all during the first six hours postoperatively. This difference in demand for rescue medication between bupivacaine and placebo was significant $(p<0.001$, Chi-square test).

There were no differences between the two groups in time to mobilization or in the time point for discharge from hospital. Superficial surgical site infections were observed only in two women before discharge from hospital. Both women received bupivacaine-adrenaline. The 
Local anesthesia and cesarean section

women's files were checked for up to a week postoperatively. Infections were treated by antibiotics. No infections were recorded at the time of a telephone interview ten days after surgery. Only five women had their dressings changed due to leakage. 


\section{Discussion}

The findings in this large single center study confirm that an injection of local anesthetics in the surgical wound is a valuable regimen for postoperative analgesia during the first 12 hours after CS performed under spinal anesthesia. The finding that women in the bupivacaine group had significantly lower morphine consumption up to twelve hours postoperatively, in addition to a small but significantly lower pain intensity score, suggests that a single injection of bupivacaine and adrenaline contributes to the quality of postoperative analgesia even when added to spinal anesthesia. Previous studies show benefits of local infiltration of local anesthetics in the surgical site but evidence regarding the benefits of a single dose of local anesthetics in the cesarean wound has been limited and contradictory. Trotter et al. found, in a 28-patient study, that bupivacaine infiltration in the surgical site reduced the amount of post cesarean rescue morphine only when adjusted for the women's weight (16). However, it is reasonable to assume that one of the reasons for the relatively minor effect observed in the study by Trotter was the small number of participants. In the present study the sample size estimation was done in order to minimize the risk of false positive or negative results related to the interdependence between morphine requirements and pain intensity. Therefore we believe that the results of the present study can be reliably interpreted.

The most common way to provide postoperative local analgesia is infiltration in the surgical wound with PCA catheters $(8,17-21)$. In a review by Bamigboye and Hofmeyr the conclusion was that local pain management decreased the postoperative consumption of morphine in women who had CS under either spinal or general anesthesia (11). Givens et al. found that the morphine consumption required to obtain good pain relief after CS was significantly lower in a group receiving local infusion of bupivacaine with a PCA catheter in the wound for 48 hours postoperatively than in a control group receiving saline (19). According to the authors, 
one weakness in the study was that the catheter itself could irritate the tissues which might lead to increased pain (19). In a similar study Fredman and coworkers suggested that catheterassociated infections could be a risk and had safety concerns about the pain pump (17). A continuous catheter technique is also expensive, and may not be possible to use as a clinical routine in all maternity clinics.

Overall, using local anesthesia for pain management can be hypothesized to decrease the risk for side-effects as nausea, vomiting and dizziness and also to decrease the risk for drugs passing over to the baby $(18,20)$. The findings in the present study demonstrate that infiltration with local anesthetics and adrenaline decreases the need of rescue opioids in the first six hours following surgery. Women treated with local injection of bupivacaine-adrenalin also needed a lower number of morphine injections. The restrictions of the mobilization of the women may be less if given a single injection in the wound compared to carrying a PCA device. Other limitations to the use of a PCA device may be the ability to use the device correctly and the considerably higher costs involved (8).

The results of the present study indicate that a single dose injection does not increase the amount of infections in the surgical site as there were only two wound infections in 260 patients. In fact, local anesthetics have an antimicrobial effect (22). In the study by Givens leakage of excess solution, leading to a need for dressing changes, was reported (19). The few leakages from injected solutions reported in our study contradict these findings. Intrathecal fentanyl and bupivacaine have rapid onset and a relatively long duration of action (1.5-2 hours). On the other hand, the duration of action with local injection of bupivacaine at the surgical site is up to six hours or longer and thus covers a significantly longer period than the spinal anesthesia, from a pain perspective the most difficult time span after the operation. In the present study there were no differences between the bupivacaine- and placebo groups with respect to demographic data. One strength of the study was that there were very few data 
missing in the first part of the study, when registering morphine consumption, pain assessment and mobilization. An exact protocol for anesthesia and pain treatment was rigorously followed with high compliance and a very low dropout rate.

Mean NRS and maximum NRS were chosen as measures to describe the women's experience of pain over time. Maximum NRS was mostly noted prior to each morphine dose, when the women were asking for extra morphine. There was a significant difference between the bupivacaine and placebo treatment groups concerning both these parameters for the first six hours, but none after this time period. The high compliance to the study protocol during study conduct is also clearly demonstrated by the similarity in pain intensity scores between groups and the difference in morphine consumption can then be considered to reflect a true difference in opioid requirements at all the analyzed time points. This further emphasizes the importance of a small group of investigators and staff working closely together in order to obtain reliable high quality study results. All women received rescue medication when they asked for it and after obtaining pain intensity assessment. Between 0-12 hours postoperatively both the significant difference in morphine consumption and in NRS assessment was found to be strongly associated with the improved pain relief obtained with a single injection with bupivacaine-adrenaline. The convincing effects of local anaesthesia demonstrated in women undergoing CS in spinal anaesthesia suggest that this regime should be confirmed for women undergoing CS in general anaesthesia, which is more common in emergency CS. In these circumstances the suggested treatment could provide an even more important contribution to pain relief, improving the conditions for effective bonding between mother and child. Several studies have also indicated that insufficient pain management after CS can lead to persistent pain. The question whether better pain relief the first six hours will affect the incidence of persistent pain will be addressed in a forthcoming study. 
Local anesthesia and cesarean section

It may be argued that there is no need for simple analgesic methods of relatively short duration of action when intravenous PCA and intrathecal injection of more long lasting opioids are available, or even continuous wound infusion. However, in its simplicity, with no necessary pump devices needed, a single local injection can provide the time needed to establish an effective oral analgesic treatment, and thus still be of clinical benefit. The development of a slow-release injectable formulation of local anesthetics would tentatively be of great benefit by prolonging the duration of action. 
Local anesthesia and cesarean section

\section{Funding}

The study was supported by a grant from the Stockholm County Council (grant no 2006023). 


\section{Legends}

\section{Table 1.}

Demographic data collected during the preoperative visit and from the patients' files. Data are presented as median and range. There were no significant differences between the two groups (Mann-Whitney U-test; $p \leq 0.05$ ).

\section{Figure 1.}

Withdrawal - flowchart

\section{Figure 2.}

The total amount of intravenous morphine (mg/patient) in the different time periods.

\section{$a$}

Significantly different from the saline group during the first six postoperative hours.

(Student's $t$-test; $p \leq 0.001$ )

b

Significantly different from the saline group during the first 12 postoperative hours.

(Student's $t$-test; $p \leq 0.001$ )

\section{Figure 3.}

Mean Numerical Rating Scale (NRS 0-10) for pain assessment during the first six postoperative hours.

a Significantly different from the saline group. 
Local anesthesia and cesarean section

(Student's $t$-test, $p \leq 0.001$ )

\section{Figure 4.}

Maximum NRS for pain assessment the first six postoperative hours.

$a$

Significantly different from the saline group.

(Student's $t$-test, $p \leq 0.001$ ) 


\section{Table}

Table 1.

\begin{tabular}{|c|c|c|c|c|c|c|}
\hline & \multicolumn{6}{|c|}{$\begin{array}{l}\text { Demographic and obstetric } \\
\text { data }\end{array}$} \\
\hline & $\mathrm{n}=$ & Bupi & in group & $\mathbf{n}=$ & Plac & group \\
\hline Age (years) & 128 & 35 & $(24-49)$ & 126 & 34 & $(21-48)$ \\
\hline Gravida & 128 & 3 & $(1-6)$ & 124 & 3 & $(1-7)$ \\
\hline Parity & 128 & 1 & $(0-4)$ & 125 & 1 & $(0-4)$ \\
\hline Previous cesarean & 128 & 1 & $(0-3)$ & 122 & 1 & $(0-3)$ \\
\hline Body Mass Index & 128 & 29.3 & $(20.3-45.3)$ & 117 & 29.9 & $(22.5-40.7)$ \\
\hline
\end{tabular}




\section{Figures and Illustrations}

Figure 1.

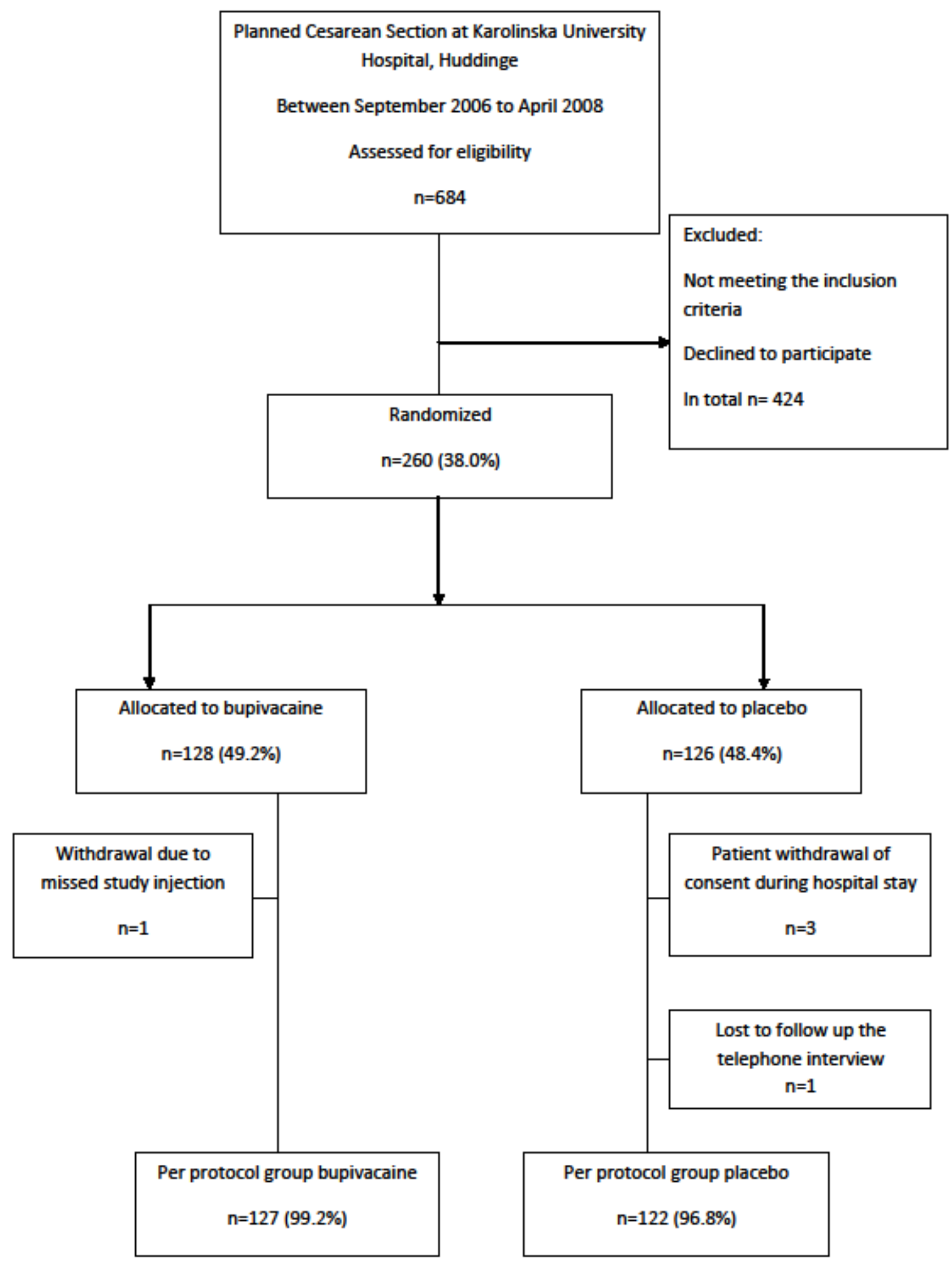


Figure 2.

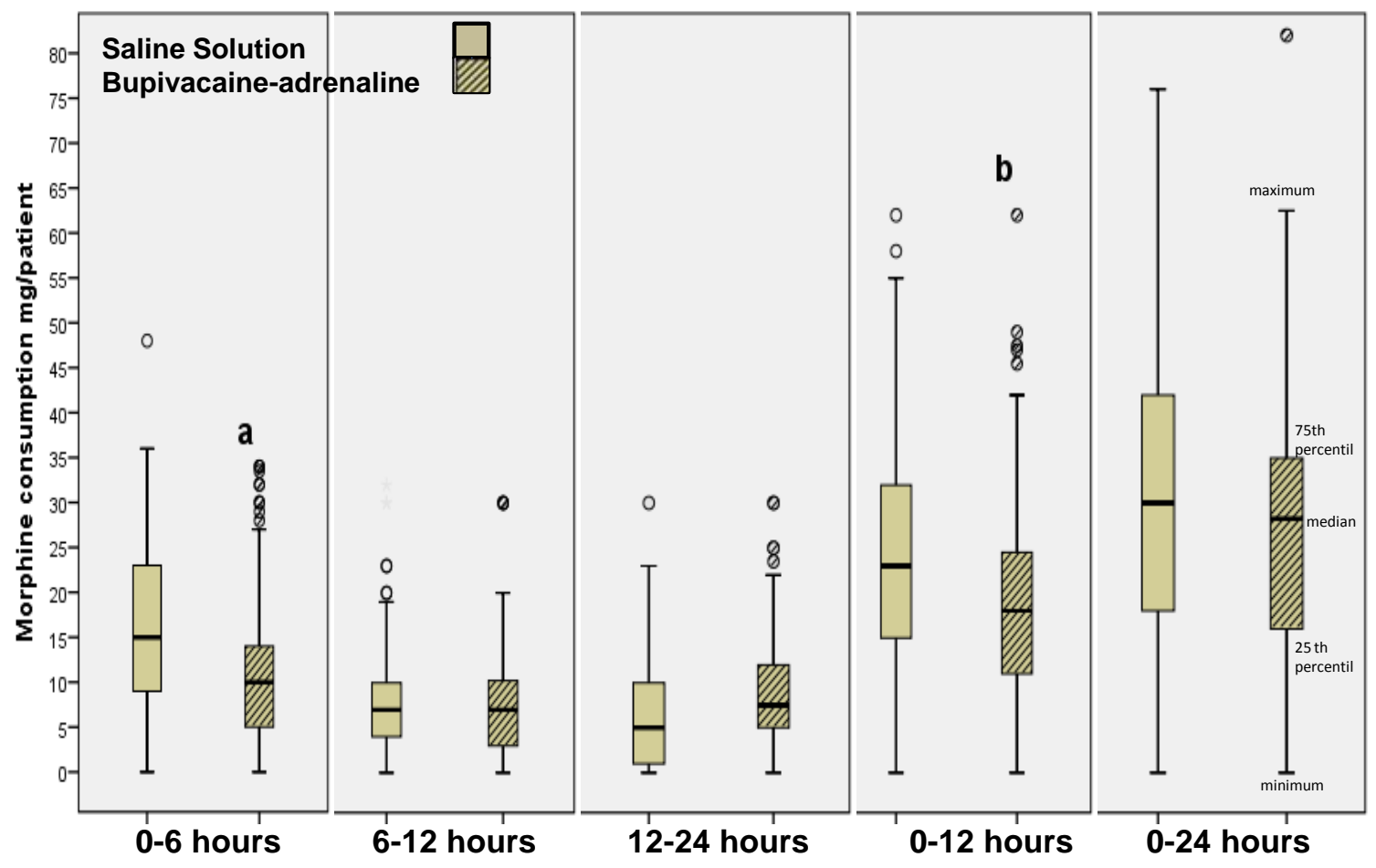


Figure 3.

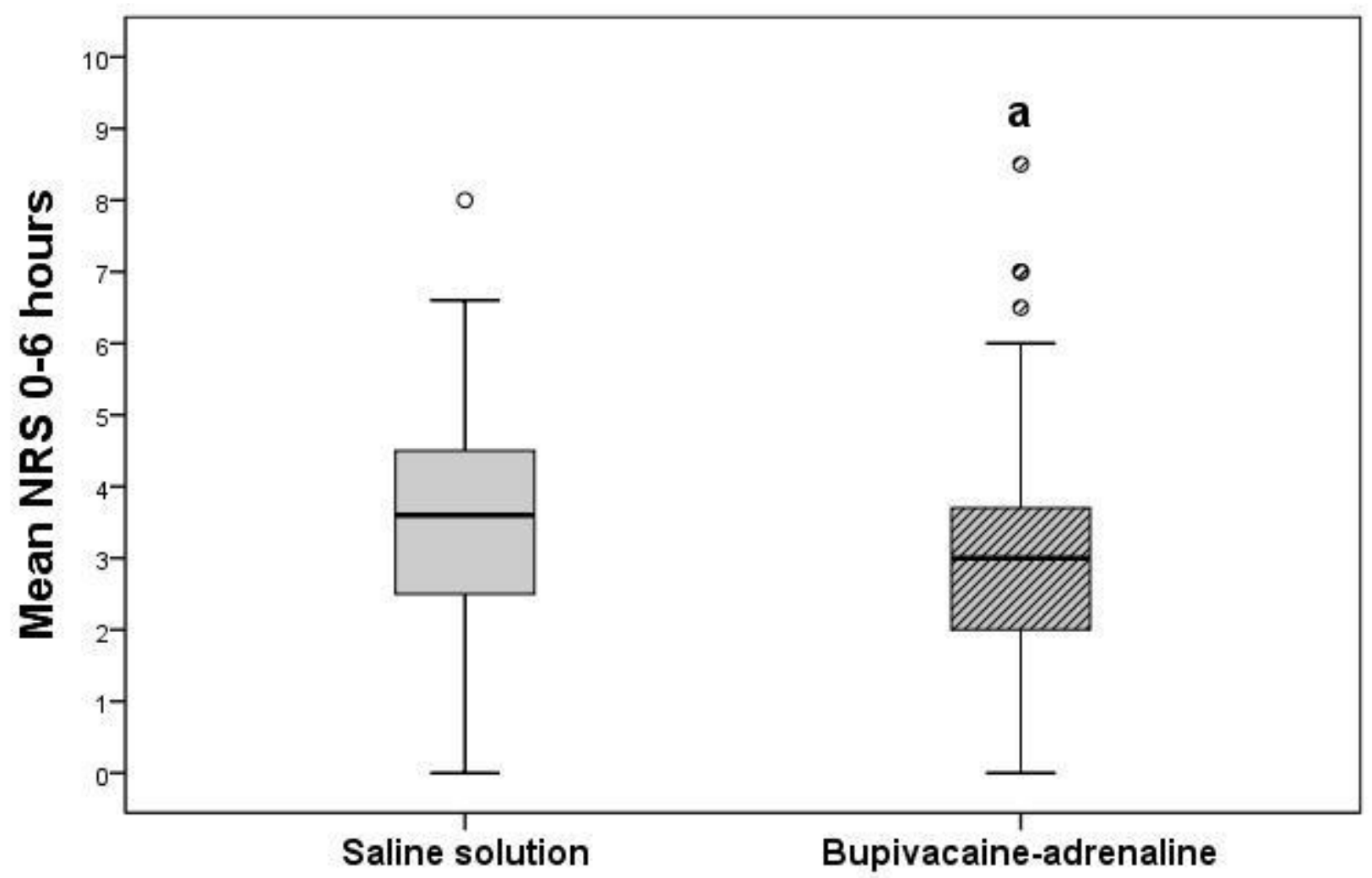


Figure 4.

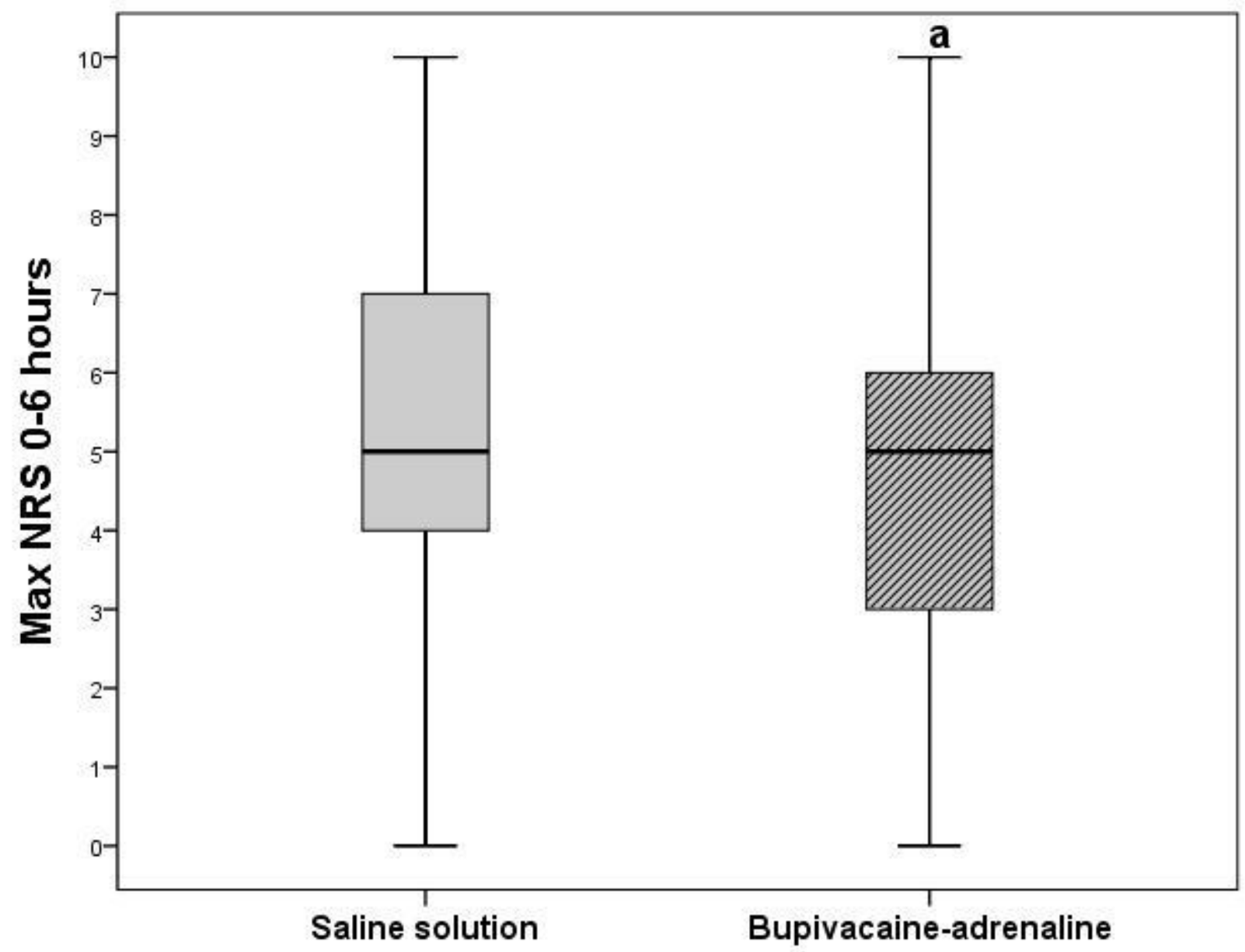

